\title{
Fatores relacionados ao prognóstico da atresia biliar pós-portoenterostomia
}

\author{
Factors related to the post-portoenterostomy prognosis of biliary atresia \\ Jorge Luiz dos Santos ${ }^{1}$, Carlos Thadeu Cerski ${ }^{2}$, Vinícius Duval da Silva ${ }^{3}$, \\ Evandro Sobroza de Mello ${ }^{4}$, Mário Bernardes Wagner ${ }^{5}$, Themis Reverbel da Silveira ${ }^{6}$
}

\section{Resumo}

Objetivo: o estudo está envolvido com o prognóstico da atresia biliar pós-portoenterostomia, presença de anomalias congênitas associadas à doença e de malformação de placa ductal, área de fibrose hepática e, sobretudo, com a idade dos pacientes por ocasião da cirurgia. O presente estudo verificou numa amostra de atresia biliar as implicações prognósticas destes fatores.

Métodos: foram avaliados 47 pacientes com atresia biliar, em estudo de corte transversal. O material histológico dos casos foi marcado com anticorpo anticitoqueratina 19 e CAM 5.2 por método imunoistoquímico, para o estudo das estruturas biliares, e corado com picrossírius para avaliação da área de fibrose. O estudo das estruturas biliares foi realizado por dois patologistas e pelo primeiro autor deste estudo, "cegos" quanto à evolução dos casos. A mensuração da área de fibrose foi quantitativa. Os dados dos pacientes em relação à idade, ocorrência de óbito ou realização de transplante hepático foram pesquisados nos prontuários.

Resultados: a idade por ocasião da portoenterostomia variou entre 24 e 251 dias de vida $(90,4 \pm 44,8$ dias), e em 32 casos ( $72 \%$ ) a evolução pôde ser acompanhada. Os 9 casos (19\%) com anomalias congênitas extra-hepáticas associadas não diferiram quanto ao prognóstico em relação ao restante da amostra. A idade por ocasião da portoenterostomia influenciou o prognóstico $(\mathrm{p}=0,016)$. A área de fibrose foi diferente entre pacientes operados com menos de 60 dias de vida e os operados com mais de 90 dias $(\mathrm{p}=0,023)$, mas não influenciou a evolução dos casos. Tampouco a presença de malformação de placa ductal influiu no prognóstico.

Conclusões: a idade por ocasião da portoenterostomia foi o único fator que afetou o prognóstico dos casos de atresia biliar. É necessário maior número de pacientes para avaliar a influência da presença de anomalias congênitas extra-hepáticas associadas sobre a evolução pósportoenterostomia.

J Pediatr (Rio J) 2002; 78(4): 341-6: atresia biliar, prognóstico pósportoenterostomia, fibrose hepática, malformação de placa ductal.

\begin{abstract}
Objective: this study considered the presence of congenital anomalies, ductal plate malformation, area of fibrosis and, mainly, the patient's age in cases of biliary atresia submitted to surgery. The present study verified the influence of these factors on the follow-up of a biliary atresia sample.
\end{abstract}

Methods: a sample of 47 patients with biliary atresia was evaluated in a cross-sectional study. Their histologic specimens were stained for antibody anticytokeratin 19 and CAM 5.2 through immunohistochemistry in order to study biliary structures, and for picrosirius red to evaluate the area of fibrosis. The study of biliary structures was performed by two pathologists and the first author of the study. They were "blind" with regard to the clinical follow-up. The area of fibrosis was quantitatively evaluated. Data on the patients with regard to age, death and occurrence of liver transplantation were searched on the patients records.

Results: age at portoenterostomy varied between 24 and 251 days of life (90.4 \pm 44.8 days) and follow-up was available in 32 cases $(72 \%)$. The nine cases $(19 \%)$ with extrahepatic congenital anomalies associated to biliary atresia did not present different prognosis from the remaining patients. Age at portoenterostomy influenced the prognosis $(p=0.016)$. The area of fibrosis was different on patients aged less than 60 days and those aged more than 90 days at portoenterostomy $(\mathrm{p}=0.023)$, but did not influence the prognosis. The presence of ductal plate malformation, as well, did not influence the follow-up.

Conclusions: age at portoenterostomy was the only factor that influenced prognosis on this sample of biliary atresia. It is necessary to increase the biliary atresia sample to check the influence of congenital extrahepatic anomalies on the follow-up postportoenterostomy.

J Pediatr (Rio J) 2002; 78 (4): 341-6: biliary atresia, postportoenterostomy prognosis, fibrosis, ductal plate malformation.

1. Doutor em Pediatria, médico contratado do Serviço de Pediatria do Hospital de Clínicas de Porto Alegre.

2. Médico contratado do Serviço de Patologia do Hospital de Clínicas de Porto Alegre.

3. Doutor em Patologia pela Universidade Federal do Rio Grande do Sul.

4. Médico contratado pelo Serviço de Patologia da Universidade de São Paulo.

5. Professor adjunto da Universidade Federal do Rio Grande do Sul.

6. Professora adjunta da Universidade Federal do Rio Grande do Sul.

Este estudo é parte da tese de doutorado do Dr. Jorge Luiz dos Santos, desenvolvida no Programa de Pós-graduação da Faculdade de Medicina da Universidade Federal do Rio Grande do Sul, na área de concentração em Pediatria.

Artigo submetido em 25.02.02, aceito em 08.05.02. 


\section{Introdução}

A atresia biliar $(\mathrm{AB})$ é uma doença exclusiva da infância, que consiste na obstrução completa de parte ou da totalidade das vias biliares extra-hepáticas. Postula-se a existência de duas formas de $\mathrm{AB}$ : congênita e adquirida ${ }^{1}$. A primeira englobaria cerca de $35 \%$ dos casos que, concomitantemente à alteração das vias biliares, apresentam anomalias congênitas extra-hepáticas associadas (ACEA), início precoce da colestase neonatal e ausência de remanescentes de ductos biliares no ligamento hepatoduodenal. A ocorrência de ACEA, pelo menos aquelas relacionadas à síndrome malformativa esplênica associada à $\mathrm{AB}$, tem sido relacionada a pior prognóstico pós-portoenterostomia ${ }^{2}$. Por outro lado, a existência de malformação de placa ductal (MPD) em cerca de $25 \%$ dos casos de $\mathrm{AB}^{3}$ foram relacionados a uma forma precoce grave da doença. A fibrose tem sido considerada expressão histológica de gravidade da $\mathrm{AB}$, e sua quantificação, nesses casos, pode ser útil na avaliação do prognóstico. Contudo, entre as diversas variáveis relacionadas à evolução pós-operatória, a idade do paciente por ocasião da portoenterostomia permanece como o fator mais importante na previsão do prognóstico ${ }^{4}$.

O presente estudo visou avaliar, em casos de $\mathrm{AB}$, as implicações prognósticas de: (1) achados histológicos, tais como a presença de MPD e extensão da fibrose; e (2) clínicos, incluindo a idade dos pacientes na portoenterostomia, e a presença de ACEA.

\section{Métodos}

Este estudo avaliou 47 pacientes com $\mathrm{AB}$, de ambos os sexos, submetidos ao protocolo de investigação de colestase neonatal no setor de gastrenterologia pediátrica do serviço de pediatria do Hospital de Clínicas de Porto Alegre, entre 1987 e 1997. Todos foram acompanhados desde a internação hospitalar até a sua alta pela mesma equipe. Parte desta amostra foi, posteriormente, atendida em ambulatório. O diagnóstico foi firmado durante a laparotomia exploradora, que introduz a cirurgia de Kasai. O delineamento do estudo foi de corte transversal, constando de uma avaliação retrospectiva e de outra prospectiva do material de biópsia hepática em cunha, fixado com formalina, incluído em parafina, obtido durante o procedimento cirúrgico. No estudo retrospectivo, incluíram-se pacientes que, ao início do estudo, já haviam sido submetidos à biópsia; no prospectivo, pacientes com as mesmas características, que foram prospectivamente atendidos a partir do início da pesquisa.

Quanto à avaliação de ACEA nos pacientes em estudo retrospectivo, foram coletadas informações nos prontuários, já que esta pesquisa é rotineiramente realizada na investigação diagnóstica da colestase neonatal. Avaliaramse dados do exame físico e de exames radiológicos, incluindo radiografia de tórax, ecografia abdominal e de sistemas específicos, além da descrição de achados clínicos, cirúrgicos e de autópsia, quando possível. No estudo pros- pectivo, houve a pesquisa de ACEA em cada paciente investigado, segundo o mesmo protocolo. Foram levadas em consideração apenas anormalidades congênitas maiores, ou seja, aquelas que afetam a viabilidade ou a fertilidade de seus portadores.

O estudo histológico das estruturas biliares foi realizado por dois patologistas e pelo primeiro autor deste estudo, "cegos" quanto à evolução clínica. Caracterizaram-se os achados quanto à presença de MPD, à presença e distribuição de ductos biliares maduros, e da proliferação ductular. A extensão da fibrose foi avaliada por método quantitativo. O prognóstico dos casos foi caracterizado a partir da evolução daqueles que mantiveram acompanhamento ambulatorial, após a realização da portoenterostomia. A avaliação do prognóstico baseou-se na ocorrência de óbito e na realização de transplante hepático (Tx) no seguimento dos casos.

As variáveis histológicas avaliadas são conceituadas a seguir.

Presença da malformação de placa ductal: caracterizada pela presença de estruturas biliares marcadas com anticorpo anticitoqueratina 19 (CK 19) ou CAM 5.2, com morfologia irregular, dilatada, formando anel circundante a tecido mesenquimatoso, com ou sem ramo de veia porta no centro $^{3}$.

Ductos biliares maduros: estruturas tubulares, circulares em corte transversal, ou alongadas em corte longitudinal, com morfologia regular, marcadas por CK 19 e CAM 5.2, localizadas no interior dos espaços-porta. Conceituouse como focal sua ocorrência em até três espaços-porta.

Proliferação ductular: definida como a presença de ductos biliares em número aumentado, localizados ao longo da placa limitante ou no interior do mesênquima. Considerou-se focal sua presença em, no máximo, três espaçosporta.

Densidade de colágeno: conceituada como a média dos valores da porcentagem de colágeno, quantificados por método morfométrico, presente em dez imagens de material histológico corado com picrossírius.

Para a preparação histológica e imunoistoquímica de cada amostra de tecido de casos com AB obtidos na data da portoenterostomia, foram utilizados quatro secções de 5 micrometros de espessura, duas das quais para reação com os anticorpos primários monoclonal CAM 5.2 (BectonDickinson) e monoclonal anticitoqueratina humana (DAKO), ambas para o estudo das estruturas biliares. A técnica imunoistoquímica utilizada foi a $\mathrm{ABC}$ peroxidase, segundo método padronizado por Hsu et al. ${ }^{5}$ Além da marcação com estes anticorpos, foi empregada a coloração por picrossírius para localização do colágeno ${ }^{6}$.

Para a avaliação da área de fibrose, utilizou-se a técnica de quantificação morfométrica da densidade de colágeno, empregada por Chevallier et al. ${ }^{7}$, a partir do material histológico corado com picrossírius, em dez imagens cap- 
tadas em dispositivo físico no formato tag image format (TIFF). Por este método, realizado em um sistema de análise computadorizada de imagens, obteve-se a quantificação automática da área de fibrose presente em um campo de superfície. A análise de imagem foi feita com o software Image-Pro ${ }^{\circledR}$ Plus, versão 4.1, da Media Cybernetics.

Em relação à análise estatística, a avaliação das diferenças entre variáveis quantitativas foi realizada através do teste t de Student, ou da análise de variância de um critério de classificação (ANOVA one-way). Para a localização de diferenças pós-ANOVA, foi adotado o procedimento de comparações múltiplas de Duncan. Nas situações de assimetria, foram utilizados os testes não-paramétricos $\mathrm{U}$, de Mann-Whitney e ANOVA, de Kruskal-Wallis. A localização de diferenças por ANOVA, de Kruskal-Wallis, foi realizada pelo procedimento de comparações múltiplas proposto por Dunn. Nos casos de variáveis qualitativas, foi utilizado o teste qui-quadrado e, quando necessário, o teste exato de Fisher. Na avaliação de associações entre variáveis ordinais e contínuas, empregou-se o coeficiente de correlação não-paramétrico, de Spearman. O nível de significância adotado foi de $\alpha=0,05$, sendo considerados de significância limítrofe os testes estatísticos que atingiram valores de $0,05<\mathrm{P}<0,1$. Os dados foram processados e analisados com o auxílio dos programas Excel versão 97, e SPSS versão 10.0 .

O presente estudo foi avaliado e aprovado pela Comissão de Ética em Pesquisa do Grupo de Pesquisa e PósGraduação do Hospital de Clínicas de Porto Alegre.

\section{Resultados}

A idade dos casos avaliados neste estudo na data da portoenterostomia variou entre 24 e 251 dias de vida, com média de 90,9 $\pm 44,8$ dias.

Nove casos (19\%) apresentaram ACEA. Não houve diferença estatística entre os grupos com e sem ACEA quanto à idade por ocasião da portoenterostomia $(\mathrm{p}=0,390)$ (dados não apresentados). A Tabela 1 avalia o prognóstico dos casos segundo os grupos com e sem ACEA.

Entre os casos em que a evolução pôde ser acompanhada pós-portoenterostomia $(\mathrm{n}=34 ; 72 \%)$, não houve diferença significante entre os dois grupos. Porém, apenas um caso com ACEA mantém-se vivo e não foi transplantado, enquanto entre os casos sem ACEA a distribuição foi mais homogênea: cerca da metade dos casos permanece vivo e não foi submetido ao Tx.

A Tabela 2 relaciona a idade por ocasião da portoenterostomia e o prognóstico.

Dezenove casos (40\% do total de casos, $56 \%$ dos pacientes em acompanhamento) faleceram ou submeteramse a Tx, enquanto 15 (32\% do total, $44 \%$ dos pacientes em acompanhamento) continuam vivos e não foram transplantados. A idade dos casos na portoenterostomia afetou o
Tabela 1 - Evolução pós-operatória dos pacientes com atresia biliar, segundo os grupos com e sem anomalias congênitas extra-hepáticas associadas

\begin{tabular}{lccc}
\hline & \multicolumn{3}{c}{ Atresia biliar } \\
Característica & $\begin{array}{c}\text { Sem anomalia } \\
\text { extra-hepática } \\
(\mathbf{n}=\mathbf{2 7})\end{array}$ & $\begin{array}{c}\text { Com anomalia } \\
\text { extra-hepática } \\
(\mathbf{n}=7)\end{array}$ & $\mathbf{p}$ \\
\hline $\begin{array}{l}\text { Mortos ou } \\
\text { transplantados }\end{array}$ & $13(48)$ & $6(86)$ & 0,103 \\
$\begin{array}{l}\text { Vivos e } \\
\text { não transplantados }\end{array}$ & $14(52)$ & $1(14)$ & \\
\hline
\end{tabular}

Porcentagem entre parênteses.

Método estatístico: teste exato de Fisher.

prognóstico de forma significante $(\mathrm{p}=0,016)$. $\mathrm{O}$ pior prognóstico ocorreu com as crianças que tinham entre 69 dias, ou mais de 90 dias, por ocasião da cirurgia.

A Tabela 3 apresenta a relação entre idade, classificada em faixas etárias, e a densidade de colágeno do material de biópsia.

Tabela 2 - Relação entre prognóstico e idade por ocasião da portoenterostomia

\begin{tabular}{lccccc}
\hline Prognóstico & N & $\begin{array}{c}\text { Idade } \\
\text { (dias de vida) }\end{array}$ & $\begin{array}{c}\text { Dife- } \\
\text { rença }\end{array}$ & IC95\% & p \\
\hline $\begin{array}{l}\text { Morto ou } \\
\text { transplantado }\end{array}$ & 19 & $97,3 \pm 35,9$ & 27,3 & 5,3 a 49 & 0,016 \\
$\begin{array}{l}\text { Vivo não } \\
\text { transplantado }\end{array}$ & 15 & $70,00 \pm 23,7$ & & & \\
\hline
\end{tabular}

Método estatístico: teste t de Student.

A densidade de colágeno nos casos de $\mathrm{AB}$, independente de faixa etária, envolveu $6,6 \% \pm 3,9 \%$ da superfície do campo histológico. Houve relação positiva entre faixas etárias ascendentes e níveis crescentes de densidade de colágeno na amostra, com diferença estatisticamente significante $(\mathrm{p}=0,023)$ entre os grupos menor ou igual a 60 dias e maior que 90 dias de vida. O valor máximo de densidade de colágeno na classe de menos de 60 dias foi de 6,3\%, enquanto que na classe de mais de 90 dias chegou a cerca de $23 \%$ do total de superfície da lâmina. A menor densidade de colágeno ocorreu em um caso da classe de 61 a 90 dias de vida $(1,7 \%)$. Neste último grupo, o valor máximo da densidade de colágeno foi de $14 \%$. Houve ainda correlação moderada positiva $(r=0,37, p=0,011)$ entre a densidade de colágeno e a idade considerada como variável contínua (dados não apresentados). 
Tabela 3 - Relação entre faixas etárias por ocasião da portoenterostomia e densidade de colágeno

\begin{tabular}{lcccc}
\hline $\begin{array}{l}\text { Faixa etária } \\
\text { dias de vida) }\end{array}$ & $\mathbf{N}$ & $\begin{array}{c}\text { Densidade de } \\
\text { colágeno }(\%)\end{array}$ & IC 95\% & p \\
\hline$\leq 60$ & 11 & $4,3 \pm 1,3^{\mathrm{a}}$ & 3,5 a $5,2^{\mathrm{a}}$ & \\
$61-90$ & 16 & $6,1 \pm 3,1$ & 4,4 a 7,8 & 0,023 \\
$>90$ & 20 & $8,1 \pm 4,7^{\mathrm{b}}$ & 5,9 a $10,3^{\mathrm{b}}$ & \\
Total & 47 & $6,6 \pm 3,9$ & 5,4 a 7,7 & \\
\hline
\end{tabular}

Letras-índice não coincidentes representam diferenças estatisticamente significativas.

Método estatístico: ANOVA.

A Tabela 4 apresenta a relação entre prognóstico e densidade de colágeno nos pacientes acompanhados pósportoenterostomia.

Não se observou relação significante entre a densidade de colágeno e o prognóstico dos casos $(\mathrm{p}=0,166)$. Observou-se grande variação da densidade de colágeno nas dez imagens aleatoriamente captadas em cada caso, o que se reflete em amplos desvios-padrão, apesar de um número razoável de pacientes envolvidos na comparação.

Tabela 4 - Relação entre prognóstico e densidade de colágeno nos pacientes com atresia biliar

\begin{tabular}{lcccc}
\hline Evolução & $\begin{array}{l}\text { Densidade de } \\
\text { colágeno (\%) }\end{array}$ & Diferença & IC95\% & p \\
\hline $\begin{array}{l}\text { Morto ou } \\
\text { transplantado } \\
(\mathrm{n}=19)\end{array}$ & $7,6 \pm 4,5$ & 1,87 & $-0,8$ a 4,6 & 0,166 \\
$\begin{array}{l}\text { Vivo não } \\
\text { transplantado } \\
(\mathrm{n}=15)\end{array}$ & $5,7 \pm 2,7$ & & & \\
\end{tabular}

Método estatístico: teste t de Student.

A Tabela 5 apresenta a relação dos achados histológicos nas estruturas biliares, e o prognóstico nos casos acompanhados.

Não foi possível identificar diferença significante quanto ao prognóstico entre casos com e sem MPD. Tampouco outros achados histológicos nas estruturas biliares influenciaram o prognóstico. Não houve, ainda, relação entre a presença de malformação de placa ductal e a idade agrupada em faixas etárias ( $\mathrm{p}=0,503)$ (dados não apresentados).

\section{Discussão}

Os dados apresentados na Tabela 1, embora não tenham demonstrado diferença significante entre os grupos de $\mathrm{AB}$ com e sem ACEA, permitiram constatar que a distribuição dos pacientes sem ACEA foi homogênea quanto ao prognóstico: cerca da metade dos pacientes morreu ou submeteu-se a Tx, enquanto $86 \%$ dos casos com ACEA estão mortos ou foram transplantados. A inexistência de relação entre a presença de ACEA e pior prognóstico neste estudo, porém, pode ter sido resultado do pequeno número de casos. Esta relação foi detectada por alguns autores ${ }^{2}$ nos casos com anomalia da seqüência de lateralidade. Para Silveira et al. ${ }^{8}$, porém, o fator prognóstico de maior relevância é a idade por ocasião da portoenterostomia. A Tabela 2 demonstra que entre 34 casos acompanhados em ambulatório, 56\% morreram ou submeteram-se a Tx até a conclusão do estudo, tendo a maioria dos pacientes com pior prognóstico sido submetida à cirurgia com mais idade. Tem sido observado fluxo biliar permanente no pós-operatório da cirurgia de Kasai, progressivamente menor com o avanço das faixas etárias ${ }^{9,10}$.

Tabela 5 - Relações entre achados histológicos nas estruturas biliares e prognóstico nos casos de atresia biliar acompanhados em ambulatório

\begin{tabular}{lccc}
\hline Variável & \multicolumn{2}{c}{ Prognóstico } & p \\
& $\begin{array}{c}\text { Morto ou } \\
\text { transplantado } \\
(\mathbf{n}=\mathbf{1 9})\end{array}$ & $\begin{array}{c}\text { Vivo não } \\
\text { transplantado } \\
(\mathbf{n}=\mathbf{1 5})\end{array}$ & \\
& & \\
\hline $\begin{array}{l}\text { Malformação de placa ductal } \\
\quad \text { Presente }\end{array}$ & $12(60)$ & $8(40)$ & 0,563 \\
$\begin{array}{l}\text { Ducto biliar maduro } \\
\quad \text { Presente }\end{array}$ & $17(90)$ & $14(93)$ & 0,693 \\
$\quad$ Focal & $4(23)$ & $4(29)$ & 0,750 \\
$\quad$ Difusa & $13(77)$ & $10(71)$ & \\
$\begin{array}{l}\text { Proliferação ductular } \\
\text { Focal }\end{array}$ & $11(58)$ & $5(33)$ & 0,154 \\
$\quad$ Difusa & $8(42)$ & $10(67)$ & \\
\hline
\end{tabular}

Método estatístico: qui-quadrado.

Porém, entre os pacientes que evoluíram satisfatoriamente no presente estudo, houve casos com mais de 60 dias de vida, e até mais de 90 dias (média $=70,0 ; \mathrm{DP}=23,7$ ), o que parece justificar a manutenção da portoenterostomia como procedimento inicial nos casos de $\mathrm{AB}$ em nosso centro hospitalar. Chardot et al. ${ }^{11}$ confirmaram melhor sobrevida em cinco e dez anos pós-Kasai em pacientes operados precocemente, mas cerca de $25 \%$ dos seus pacientes operados após noventa dias de vida sobreviveram com seu próprio fígado por cinco anos, e cerca de $22 \%$ por dez anos. Esse achado justificaria, segundo eles, a manutenção desse procedimento cirúrgico mesmo em pacientes com 
mais de três meses de idade. No presente estudo, cerca de $32 \%$ dos pacientes submetidos à cirurgia de Kasai ( $44 \%$ dos que mantêm acompanhamento ambulatorial), independentemente da idade em que foram operados, permanecem vivos e não foram transplantados. Na literatura internacional, essa freqüência chega a $42 \%$ em cinco anos ${ }^{10}$. Grosfeld et al. ${ }^{12}$ constataram influência das variáveis idade na portoenterostomia e obtenção de fluxo biliar no pós-operatório, em relação à sobrevida. Reforçaram a noção de que a portoenterostomia deva ser mantida como procedimento inicial nos casos de $\mathrm{AB}$, evitando-se a morbidade associada ao Tx. Ohya et al. ${ }^{13}$ constataram que a sobrevivência em dez anos pós-portoenterostomia relacionou-se ao desaparecimento da icterícia no pós-operatório, à idade na cirurgia, ao grau de fibrose e ao tamanho dos ductos biliares no porta hepatis. No entanto, Tan et al. ${ }^{14}$ não encontraram relação entre a idade dos pacientes na portoenterostomia e a presença de ductos biliares pérvios no porta hepatis. Afirmaram que a idade é importante apenas em relação aos casos operados com menos de 60 dias de vida. Outros autores $^{15,16}$, ainda, não encontraram relação entre a idade e o prognóstico pós-Kasai. Deve-se levar em conta que a relação entre a idade e o prognóstico pós-portoenterostomia não é linear, e que o efeito do atraso da cirurgia só tornase óbvio após um limiar de tempo. Grupos de pacientes com idade abaixo desse limiar não apresentarão relação entre faixa etária e evolução ${ }^{17}$. Maksoud et al. ${ }^{18}$ observaram drenagem biliar pós-portoenterostomia em cerca de $73 \%$ das crianças e ausência de icterícia em $27 \%$, aproximadamente. Até 16 semanas de vida, não houve relação entre idade e fluxo biliar no pós-operatório; após esse limite, o fluxo biliar decaiu, embora permanecendo em quase metade dos pacientes.

Quanto à extensão da fibrose, observou-se aumento progressivo em relação às faixas etárias, havendo diferença significante entre os grupos de até 60 dias de vida e o de mais de 90 dias de vida (Tabela 3). Aparentemente, a faixa etária de mais de 90 dias relacionou-se a um limiar na intensidade da fibrose. $\mathrm{O}$ fato de não termos encontrado relação entre prognóstico e a densidade superficial de colágeno (Tabela 4) concorda com os achados de Tan et al. ${ }^{14}$ : fibrose no porta hepatis de todos os casos e ausência de relação entre a morfologia da árvore biliar periférica, incluindo fibrose, e o prognóstico. Schweizer et al. ${ }^{19}$, embora encontrando relação positiva entre extensão da fibrose e pior evolução, consideraram-na como um critério secundário na determinação do prognóstico na $\mathrm{AB}$.

Conforme os dados demonstrados na Tabela 5, não houve relação entre a presença de MPD e pior prognóstico pós-cirúrgico, contrariando a afirmação de Desmet $^{3}$, de que os pacientes com esta anomalia representariam uma forma precoce grave da doença.

$\mathrm{A} \mathrm{AB}$ relaciona-se à progressiva destruição de ductos biliares interlobulares após os dois meses de vida. $\mathrm{Na}$ maioria das crianças com $\mathrm{AB}$, a doença hepática progride independentemente à desobstrução do fluxo biliar ${ }^{20}$, talvez em função dos episódios recorrentes de colangite que agravam a fibrogênese e/ou da existência de um processo mórbido continuado que mantém a degradação funcional e histológica do fígado ${ }^{21}$. Há um padrão anômalo da árvore biliar na AB, o qual pôde ser melhor compreendido após as considerações sobre a presença de MPD na doença ${ }^{3}$. Outro dado relevante quanto ao mau prognóstico de muitos pacientes após a cirurgia de Kasai, é a presença precoce de hipertensão porta ${ }^{22}$.

A maioria dos pacientes, após a cirurgia de Kasai, desenvolve fibrose hepática progressiva. Em cerca de 1/3 dos casos ocorre insuficiência hepática, levando a Tx em 12 a 14 meses de pós-operatório, e em outro $1 / 3$ durante a adolescência. Os restantes sobrevivem com algum grau de hepatopatia ${ }^{23}$. No presente estudo, portanto, apenas a idade na portoenterostomia influenciou o prognóstico pós-cirúrgico. A extensão da fibrose e as alterações histológicas das estruturas biliares, incluindo MPD, na periferia hepática, não apresentaram relação com pior prognóstico. Seria necessário um maior número de casos para considerar a relação entre a existência de ACEA e uma pior evolução após a portoenterostomia. A média de idade dos pacientes na portoenterostomia mantém-se elevada em nosso centro hospitalar em relação à avaliação anterior ${ }^{4}$, demonstrando que o encaminhamento dos pacientes com $\mathrm{AB}$ permanece tardio. A AB deveria ser, no entanto, considerada pelos pediatras de nossa comunidade uma urgência médica.

\section{Referências bibliográficas}

1. Balistreri W, Grand R, Hoofnagle J, Suchy F, Ryckman F, Perlmutter D, et al. Biliary atresia: current concepts and research directions - summary of a symposium. Hepatology 1996;23: 1682-92.

2. Davenport M, Savage M, Mowat A, Howard E. Biliary atresia splenic malformation syndrome: an etiologic and prognostic subgroup. Surgery 1993;113:662-8.

3. Desmet V. Congenital diseases of intrahepatic bile ducts: variations on the theme "Ductal Plate Malformation". Hepatology 1992;16:1069-83.

4. Santos J, Silveira T, Almeida H, Carvalho P, Cerski C. Colestase neonatal-atraso no encaminhamento de crianças para diagnóstico diferencial. J Pediatr (Rio J) 1997;73:32-6.

5. Hsu S, Raine L, Fanger H. Use of avidin-biotin-peroxidase complex $(\mathrm{ABC})$ in immunoperoxidase techniques: a comparison between $\mathrm{ABC}$ and unlabelled antibody (PAP) procedures. J Histochem Cytochem 1988;8:1586-95.

6. Junqueira L, Bignolas G, Brentani R. Picrosirius staining plus polarization microscopy, a specific method for collagen detection in tissue sections. Histochem J 1979;11:447-51.

7. Chevallier M, Guerret S, Chossegros P, Gerard F, Grimaud J. A Histological semiquantitative scoring for evaluation of hepatic fibrosis in liver needle biopsy specimens: comparison with morphometric studies. Hepatology 1994;20:349-55.

8. Silveira T, Salzano F, Howard E, Mowat A. Congenital structural abnormalities in biliary atresia: evidence for etiopathogenic heterogeneity and therapeutic implications. Acta Paediatr Scand 1991;80:1192-99. 
9. Questa H, Valone P, Wacholder V. Atresía de vias biliares. Seguimos operando tarde? Revista de Cirugía Infantil 1993; 3:118-51.

10. Rudolph J, Balistreri W. Optimal treatment of biliary atresia "halfway" there! Hepatology 1999;30:808-10.

11. Chardot C, Carton M, Spire-Bendelac N, Le Pommelet C, Golmard J, Reding R, et al. Is the Kasai operation still indicated in children of more than 3 months diagnosed with biliary atresia? J Pediatr 2001;138:224-8.

12. Grosfeld J, Rescorla F, Skinner M, West K, Scherer III L. The spectrum of biliary tract disorders in infants and children. Arch Surg 1994;129:513-20.

13. Ohya T, Miyano T, Kimura K. Indication for portoenterostomy based on 103 patients with Suruga II modification. J Pediatr Surg 1990;25:801-4.

14. Tan C, Davenport M, Driver M, Howard E. Does the morphology of the extrahepatic biliary remnants in biliary atresia influence survival? A review of 205 cases. J Pediatr Surg 1994;29:1459-64.

15. Carceller A, Blanchard H, Alvarez F, Dickens S, Bensoussan A, Di Lorenzo M. Past and future of biliary atresia. J Pediatr Surg 2000;35:717-20.

16. Schoen B, Lee H, Sullivan K, Ricketts R. The Kasai portoenterostomy: when is it too late? J Pediatr Surg 2001;36:97-9.

17. McKiernan P, Baker A, Kelly D. The frequency and outcome of biliary atresia in the UK and Ireland. Lancet 2000;355:25-9.

18. Maksoud J, Fauza D, Silva M, Porta G, Miura I, Zerbini M. Management of biliary atresia in the liver transplantation era: a 15-year, single-center experience. J Pediatr Surg 1998;33:115-8.
19. Schweizer P, Schweizer M, Schellinger K, Kirschner H, Schittenhelm C. Prognosis of extrahepatic bile-duct atresia after hepatoportoenterostomy. Pediatr Surg Int 2000;16:351-5.

20. Lilly J, Altman R. Hepatic portoenterostomy (the Kasai operation) for biliary atresia. Surgery 1975;78:76-86.

21. Landing B. Considerations of the pathogenesis of neonatal hepatitis, biliary atresia and choledochal cyst - the concept of infantile obstructive cholangiopathy. Prog Pediatr Surg 1974;6:113-39

22. Ohi R, Mochizuki I, Komatsu K, Kasai M. Portal hypertension after successful hepatic portoenterostomy in biliary atresia. $\mathbf{J}$ Pediatr Surg 1986;21:271-4.

23. Ramm G, Nair V, Bridle K, Shepherd R, Crawford D. Contribution of hepatic parenchymal and nonparenchymal cells to hepatic fibrogenesis in biliary atresia. Am J Pathol 1998;153:527-35.

Endereço para correspondência:

Rua Fernandes Vieira, 501 - ap. 02 - Bom Fim

CEP 90035-091 - Porto Alegre, RS

E-mail: kapars@zipmail.com.br 